\title{
Maxwell equations and the properties of spatial-temporary continuum
}

\section{Stepanova T.R., Vahhi E.N.}

Peter the Great St.Petersburg Polytechnic University, St.Petersburg, Russia.;

E-mail: Stepanova <uranova.marina@yandex.ru>;

This work is an investigation of the properties of space-time continuum. The phase velocity of the wave is a characteristic of the medium in which the wave propagates. Velocity of the electromagnetic wave is an invariant in all inertial frames of reference. This postulate of the relativistic theory is a characteristic of space-time.

Diffraction phenomenon is one of the fundamental features of the wave process. Formal (mathematical) theory of diffraction uses vector algebra and Fourier - transform. Real diffraction pattern exists in the dual (conjugate) space. Therefore: 1) Discrete space is a limited space, and continuity of space is infinity. 2) Vector of space-time and momentum-energy are mutually conjugate vectors. Conjugation of vectors of coordinates and momentum, and also time and energy appears as Heisenberg's uncertainty principle.

Keywords: Space, time, Maxwell equations, conjugate vectors, Fourier - transform, Fraunhofer diffraction. DOI: $10.18698 / 2309-7604-2015-1-502-510$

\section{Introduction}

The relativity principle can be formulated as a statement, that the laws of nature are invariant with regarding transformations of movement. Also, other principles of invariance and symmetry are known from practice. It is known proof of direct and inverse theorems, that homogeneity of space $=$ the law of conservation of momentum, the isotropy of space $=$ the law of conservation of angular momentum, the homogeneity of time = the law of energy conservation. These four transformation form a group called the Poincare group. Transformations of the Poincare group are universal. So, we may assume, that Poincare group describes the properties of space-time, rather than the properties of specific processes [1].

The wave motion is periodic. Function $\vec{E}(t, \vec{r})=\vec{E}_{0} \exp (i(\omega t-\vec{k} \vec{r}))$ describes a plane wave. Its argument is a linear combination of time and coordinates, which is a phase of the oscillations. If we are moving along the oscillation curve both on the time scale and the scale of the coordinates, we observe that these fluctuations are similar. From this viewpoint, the spatial and temporal coordinates are indistinguishable. When we integrate wave space (electromagnetic field) and classical physics we get the electromagnetic constant, which binds the position and time. It's the speed of light $\mathrm{c}=\frac{\omega}{\mathrm{K}}$. So, we can say that the SRT is an extension of classical mechanics, i.e. it's the union of classical mechanics and electrodynamics. But this model is not sufficient. The 
quantum mechanics appears to explain the phenomena that could not be explained by electrodynamics. So the problems, which associated with the properties and structure of space, are very interesting. In [2] there is very effective concept which reduces the known properties of matter (wave and corpuscular) to the space -time structure. In addition the founders of the relativity theory and quantum mechanics considered matter and its properties as a product of space [3].

The appearance of the SRT was caused by conflict of electromagnetism laws and Newton laws. In the inertial reference frames there is force, which is not invariant under Galilean transformations. So, the Galilean transformations were replaced by Lorentz transformations. Contradiction was eliminated. And the fundamental concept of space-time have changed.

\section{Oscillations in mechanics and Maxwell equations.}

The wave formula is a solution of the Maxwell equations, it means that the wave equation is incorporated therein. Maxwell equations describe all the known properties of the electric and magnetic fields, and e / $\mathrm{m}$ wave is a part of them. In [4] the author analyzes the Maxwell equations and interprets them in terms of mechanics. Usually, these equations look like

$$
\begin{array}{ll}
\nabla \cdot \vec{D}=\rho & \nabla \cdot \vec{B}=0 \\
\nabla \times \vec{E}=-\frac{\partial \vec{B}}{\partial t} & \nabla \times \vec{H}=\vec{j}+\frac{\partial \vec{D}}{\partial t}
\end{array}
$$

It's possible to get the modified equations, and then we'll compare them with the equations of the theory of elasticity.

Let us suppose that

$$
\vec{E}=-\frac{1}{c} \frac{\partial \vec{u}}{\partial t}, \vec{B}=\nabla \times \vec{u}, \vec{u}=\nabla \varphi+\nabla \times \vec{\Phi}, \nabla \vec{\Phi}=0, \vec{j}=\nabla \varphi^{*}+\nabla \times \vec{\Phi}^{*}, \varphi^{*}=\frac{\varepsilon_{0}}{c} \frac{\partial^{2} \varphi}{\partial t^{2}}, \nabla \vec{\Phi}^{*}=0
$$

we obtain

$$
\Delta \vec{\Phi}-\frac{1}{c^{2}} \frac{\partial^{2} \vec{\Phi}}{\partial t^{2}}+\frac{1}{\varepsilon_{0} c} \Phi^{*}=0 \quad, \quad \Delta \varphi=q, \frac{\partial q}{\partial t}=-\frac{c \rho}{\varepsilon_{0}}
$$

where $\vec{u}$ - the vector potential, $\vec{j}$ - current density, $\rho$ - charge density, $q$ - charge. 
As for the elastic or mechanical waves, we know that these waves are longitudinal and transverse. After comparison we see, that the electromagnetic wave can be longitudinal, but its velocity should be equal to $\infty$. The author [4] points on this fact. We are going to pay attention to another fact. The existence of waves depends on the properties of matter. We know that in a vacuum elastic waves do not exist. In a solid, there are longitudinal and transverse elastic waves in the gas - only longitudinal. The velocity of propagation of elastic waves depends on the elastic properties of matter. Propagation speeds of longitudinal or transverse waves are different. Movement of the receiver or the transmitter does not affect to the properties of matter. Thus, the Doppler effect changes the frequency of the wave, and does not change the speed of its distribution. In the vacuum continuum only transverse electromagnetic waves spread and velocity of wave propagation is $c=2,998 \cdot 10^{8} \mathrm{M} / \mathrm{c}$. In a dielectric speed of electromagnetic wave is different. And offer in an anisotropic dielectric general direction of wave propagation $\vec{k}$ and the direction of energy transfer $[\vec{E} \times \vec{H}]$ are different. We can say that constant c is characteristic of electromagnetic permeability of vacuum. It is fixed in the International System of Units (SI).

\section{Wave properties of space-time continuum}

The fundamental properties of space-time continuum are described by wave processes. Let's consider wave diffraction on some object. Diffraction was defined by Zommerfeld as «... any deviation from the straight light path, besides which can be interpreted as a reflection or refraction. " If we consider the interaction of wave and medium at the atomic level, the reflection, refraction and diffraction are the result of the wave scattering. Electromagnetic waves are scattered by electrons (atomic shells). The de Broglie electron wave is scattered by the electrostatic potential of the atoms, similar wave of neutrons is scattered by the nucleus and the magnetic moment of the atom. Therefore, the terms "diffraction" and "scattering" are often used interchangeably.

Wave function $\Psi(\vec{r}, t)$ describe the harmonic plane wave. It's form is:

$$
\Psi(\vec{r}, t)=a \exp (i(\omega t-\vec{k} \vec{r}))
$$

Where $a$ - amplitude: $\vec{k}$ - the wave vector: $\vec{r}$ - coordinate of the observation point; $\mathrm{t}$ time.

The constant phase surface, where $\vec{r} \cdot \vec{k}=$ const is a perpendicular plane to the vector $\vec{k}$. 
For a spherical harmonic wave

$$
\Psi(\vec{r}, t)=\psi(r, t)=\frac{a}{r} \exp (i(\omega t-\vec{k} \vec{r}))
$$

where $r=|\vec{r}|$. In this case, the wave surface is the sphere surface. Sphere radius is $r$. Let us select the phase factor depended only on the coordinates, It's designated $\varphi(\vec{r})$. For a plane wave we obtain

$$
\begin{gathered}
\Psi(\vec{r}, t)=\Psi_{0}(\vec{r}) \exp (i \omega t), \text { where the } \\
\Psi_{0}(\vec{r})=a \exp (-i \vec{k} \vec{r})=a \exp (-i \varphi(\vec{r}))-\text { complex amplitude. }
\end{gathered}
$$

Let's find the amplitude of the diffracted waves at the point M, which is in the Fraunhofer zone. The complex amplitudes, because of different scattering centers, are added. We take into account only an optical path difference $\Delta=\left(\vec{e}-\vec{e}_{0}\right) \cdot \vec{r}$.

$$
\begin{gathered}
A(M)=a_{0} \sum_{j=o}^{N-1} \exp \left(-i \varphi_{j}\right), \\
\varphi_{j}=\frac{2 \pi}{\lambda}\left(\vec{e}_{j}-\vec{e}_{0}\right) \vec{r}=\frac{2 \pi}{\lambda} \Delta \vec{e}_{j} \vec{r} .
\end{gathered}
$$

Let, $\rho(\vec{r})$ the space density of scattering centers. Hence:

$$
A(M)=a_{0} \int \rho(\vec{r}) \exp (-i \Delta \varphi) d V, \text { where } \Delta \varphi=\frac{2 \pi}{\lambda} \Delta \vec{e}_{\varphi} \cdot \vec{r}
$$

The amplitude of the diffracted wave in the Fraunhofer zone is the Fourier transformation of the density of scattering centers $\rho(\vec{r})$.

Let's consider the formation of the optical image in the approximation of geometrical optics (Figure 1). We see dimensional object with a transmittance $\mathrm{f}(\mathrm{x})$. Left plane monochromatic 
wave with amplitude $a_{0}$ falls on the object. Just behind the object the amplitude of the scattered wave is equal $a_{0} f(x)=A_{0}(x)$.

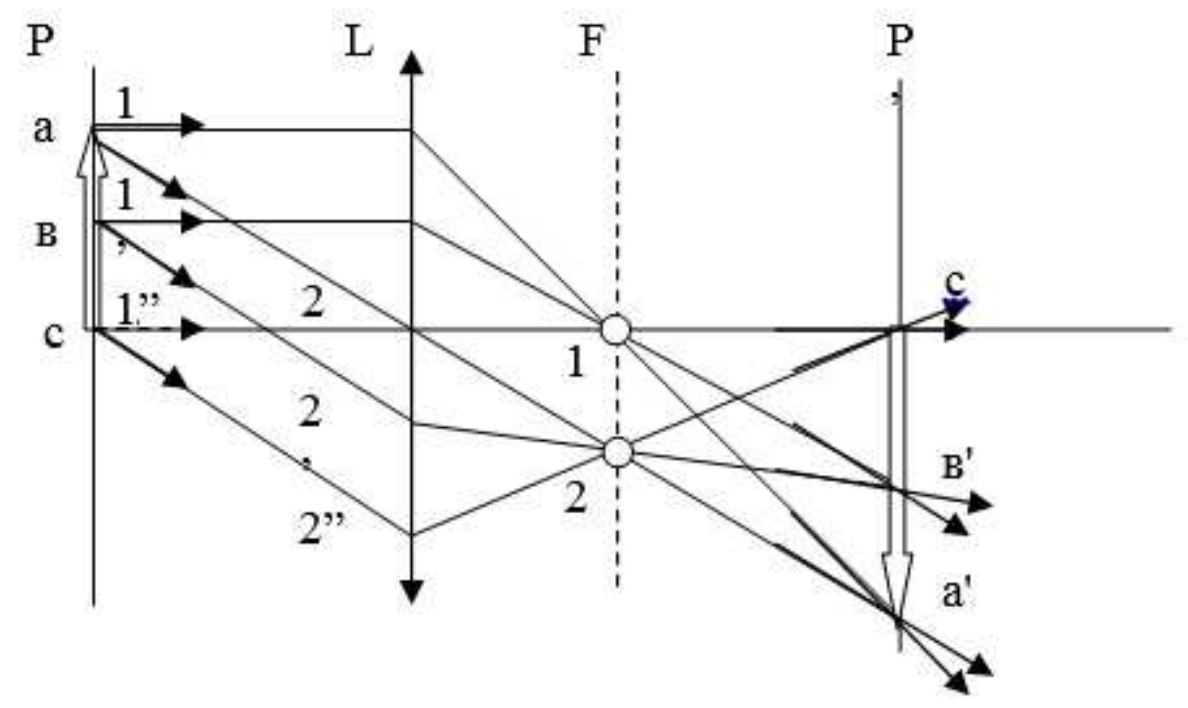

Fig. 1. Image formation in the geometrical optics approximation.

Let's consider the rays $\left.\left(1,1^{\prime}, 1^{\prime \prime}\right)=1\right\}$ and $\left\{\left(2,2^{\prime}, 2^{\prime \prime}\right)=\{2\}\right.$. If we put a screen in the Fraunhofer zone, the rays $\{1\}$ and $\{2\}$ will give points 1 and 2 on the screen. All of these points form a diffraction pattern (Fraunhofer spectrum). In Figure 1 the Fraunhofer spectrum is moved by lens $\mathrm{L}$ from infinity to focal plane $\mathrm{F}$. The amplitude of the wave in this plane is written as $A_{\Phi}=a_{0} F[f(x)]$ where the $F$ - Fourier transform symbol. The next step of image formation is a Fourier transformation of the diffraction pattern, placed in the focal plane. Thus we obtain the amplitude in the image plane $A_{P}\left(x^{\prime}\right)=a_{0} F[F[f(x)]]=a_{0} f\left(-k x^{\prime}\right)$. The coefficient $k$ is determined by the properties and lens location relative to the object. Image of the object is the result of two Fourier transformations. In a case of Fraunhofer diffraction we can obtain the first transformation without the lens. Is it possible to obtain image which is the result of the inverse Fourier transformation without a lens? It is holography. At first, we create a first Fourier transform of the object on a physical medium. Then, we illuminate holographic plate by the same spectrum light. So we get two images: real, inverted and imaginary, regular. The information about the complex amplitude is needed for reconstruct the image of object, i.e. information about the phase and amplitude. 
Let's consider the metric of the diffraction pattern. It can be seen from Fig. 1, the rays of the type $\{1\}$ emanate from different object points and fall at the point (1) in the diffraction pattern as well as the scattered rays $\{2\}$ fall at point (2). In other words, the unique connection between the object space coordinates and the coordinates of the Fourier space does not exist. Each of the points of the Fourier space gets information from all points of the object, as well as each of the object points transmits information to all points of the Fourier space.

Is the Fourier space real? [5]. If we consider the Fraunhofer diffraction, the space, where we register the diffraction pattern on photo plate, is real as well as the object space. Counter Diffractometer counter registers scattered photons, "catches" them out of the Fourier space. Spacetime continuum contains a "usual" space of object and "reverse" Fourier space.

If a function $\mathrm{f}(\mathrm{x})$ exists in the interval $\left(x_{1}, x_{2}\right)$. Let's define period as $T=x_{2}-x_{1}$ and the frequency as $v=n v_{0}=\frac{n}{T}$. Hence the function can be represented as

$$
f(x)=\sum_{v=-\infty}^{\infty} C(v) \exp (i 2 \pi v x)
$$

where the

$$
C(v)=\frac{1}{T} \int_{-T / 2}^{T / 2} f(x) \exp (-i 2 \pi v x) d x
$$

We denote $T C(v)=F(v)$ and then period $\mathrm{T}$ is tended to infinity. Hence

$$
\begin{aligned}
& f(x)=\int_{-\infty}^{\infty} F(v) \exp (-i 2 \pi v x) d v \\
& F(v)=\int_{-\infty}^{\infty} f(x) \exp (-i 2 \pi v x) d x .
\end{aligned}
$$


Equation (3) describes the function $f(x)$ in the spatial domain; the equation (4) describes the function in "frequency" domain. We can restore $f(x)$, using the relation (3) and coefficients of $F(v)$. From (1) and (3) we see that the vector $\frac{\Delta \vec{e}_{\varphi}}{\lambda}$ is the vector of spatial frequency $\vec{v}$. The dimension of the vector $\vec{v}$ is the inverse of dimension of the vector $\vec{r}$.

Using the basis of the general form $\left\{\vec{a}_{i}\right\}$, we write the vector $\vec{r}$ in object space as:

$$
\vec{r}=\vec{a}_{1} r^{1}+\vec{a}_{2} r^{2}+\vec{a}_{3} r^{3}=\vec{a}_{i} r^{j},
$$

and the vector $\vec{v}$ in Fourier space as

$$
\vec{v}=v_{1} \vec{a}^{1}+v_{2} \vec{a}^{2}+v_{3} \vec{a}^{3}=v_{i} \vec{a}^{i}
$$

There is simple correspondence between the bases. It's the normalization condition:

$$
\vec{a}_{i} \cdot \vec{a}^{j}=\lambda \delta_{i}^{j}
$$

The vectors of the main basis $\left\{\vec{a}_{i}\right\}$ are oriented relative to the vectors of the conjugate basis

$$
\left\{\vec{a}^{i}\right\}: \vec{a}_{i} \perp \vec{a}^{j}, \vec{a}^{k} \text { и } \vec{a}^{i} \perp \vec{a}_{j}, \vec{a}_{k}, i, j, k=1,2,3
$$

Object space and space of Fourier image are conjugated due to the symmetry of normalization condition regarding

In the canonical basis: $\vec{i}, \vec{j}, \vec{k}$. One denote $\left\{\vec{e}_{i}\right\}: \vec{i}=\vec{e}_{1}, \vec{j}=\vec{e}_{2}, \vec{k}=\vec{e}_{3}$, Hence $\left|\vec{e}^{i}\right|=1, \vec{e}^{i} \| \vec{e}_{i}$ and $\vec{e}_{i} \cdot \vec{e}^{j}=\delta_{i}^{j}$.

Triple of vectors $\left\{\vec{e}^{i}\right\}$ have the same magnitude and direction as the triple of vectors $\left\{\vec{e}_{i}\right\}$. However the properties of the object space and Fourier space are different. 
The metric describing by the metric tensor $\hat{g}$, is the same metric in both spaces. When operating in the object space it is necessary to use covariant coordinates of tensor $\left\{g_{i j}\right\}$, And, when operating in image space it's necessary to use contravariant coordinates of this tensor $\left\{g^{i j}\right\}$. These coordinates are given as

$$
g_{i j}=\vec{a}_{i} \cdot \vec{a}_{j} ; g^{i j}=\vec{a}^{i} \cdot \vec{a}^{j}
$$

The relation between the vectors can be written as $r_{i}=g_{i j} r^{j} ; r^{i}=r_{j} g^{j i}$

\section{Conclusion}

Relativity theory is a theory of existence and of the motion of matter in space and time. Relativity theory is the foundation of the theory of gravity and the theory of elementary particles. [6]. The moving elementary particles possess corpuscular - wave dualism. They can be described as the de Broglie wave. The development of the space - time concepts will improve the understanding of the microcosm nature. The following provisions are highlighted in this article. The phase wave speed is a characteristic of the medium in which the wave propagates. Velocity of the electromagnetic wave is invariant in all inertial frames. This postulate of the relativity theory is a characteristic of space - time continuum.

Diffraction phenomenon is one of the fundamental peculiarities of the wave process. In formal (mathematical) theory of diffraction they use vector algebra and Fourier - transforms. Real diffraction pattern exists in the dual (conjugate) space. Hence, the discreteness of space means limitation of space and continuity of space means infinity of space. And then description of the motion of objects is possible by space-time coordinates $(x, y, z, t)$ in four-dimensional space-time of Minkowski , They correspond to the invariant $s^{2}=c^{2} \Delta t^{2}-\Delta x^{2}-\Delta y^{2}-\Delta z^{2}$ And it is possible - by vector of momentum - energy $\left(p_{x}, p_{y}, p_{z}, \mathrm{E}\right)$, It corresponds to the invariant $\mathrm{E}^{2}-c^{2}\left(p_{x}^{2}+p_{y}^{2}+p_{z}^{2}\right)=m^{2} c^{4}$.Thus, we can-assume that the vector of space-time and momentumenergy are mutually conjugate. Conjugation of coordinate and momentum, as well as time and energy is manifested in the form of the uncertainty relation (Heisenberg). 
We looked at the properties of wave processes, which are determined by the properties of space. Rotational - vibrational motion of matter there is a global phenomenon. Investigation of wave processes can be useful in studying the properties of space - time.

\section{References}

1. Fizicheskij jenciklopedicheskij slovar' [Physical encyclopaedic dictionary]. (1983). Moscow: Sov. Jenciklopedija [Sov. Encyclopedia].

2. Deutch D. (2001). The structure of reality. Izhevsk: NIC "Reguljarnaja i haoticheskaja dinamika" [ SRC "Regular and chaotic dynamics"].

3. Born M. (1977). Thoughts and memories of physics. Moscow: Nauka [Science].

4. Zhilin P.A. (1997). Classical Electrodynamics and modified. Problems of space, time, motion, Proceedings of the IV International Conference dedicated to the 400th anniversary of Descartes and Leibniz's 350th anniversary, Vol. II, 29 -42.

5. Vasiliev D.M. (1998). Diffraction methods of research structures. Textbook for high schools. St. Petersburg: SPbGTU.

6. Gladyshev V.O., Kauc V. L. (2011). On the further development and expansion of the relativity theory. "Physical interpretations of relativity theory" Journal of Bauman MGTU, Moscow: BMSTU, $5-14$. 